\title{
Motivación para el Estudio y la satisfacción con la Imagen Corporal de los estudiantes de 4to y 5 to de secundaria de un Colegio en Santa Anita
}

\author{
Motivation for the Study and satisfaction with the Body Image of 4th and \\ 5 th year high school students of a school in Santa Anita
}

\author{
Susana Vigo Torres ${ }^{1}$ \\ Colegio San Francisco de Borja
}

Recibido: $03-04-18$

Aceptado: $23-07-18$

\begin{abstract}
Resumen
La actual investigación tuvo como objetivo dar a conocer las relaciones existentes entre la motivación para el estudio y la imagen corporal de los estudiantes pertenecientes a una Institución Educativa Privada en el distrito de Santa Anita (Lima-Perú). La investigación tuvo un diseño descriptivo correlacional tomando una muestra de 210 alumnos de 4 to y 5 to de educación secundaria de un mismo colegio, a quienes se aplicó dos instrumentos, Cuestionario de Motivación y Estrategias de Aprendizaje y el Cuestionario para la Evaluación de la Insatisfacción con la Imagen Corporal (IMAGEN). Los resultados nos revelan que existen correlaciones significativas respecto a la motivación al estudio y a la imagen corporal de los estudiantes de la Institución mencionada. Las dimensiones de Motivación y Estrategias de Aprendizaje se relacionan en su mayoría con los factores de Evaluación de la Insatisfacción con la Imagen Corporal, exceptuando la dimensión Atribución interna del éxito que no se relaciona.
\end{abstract}

Palabras Clave: Estudiantes; institución educativa privada; motivación; satisfacción con la Imagen corporal.

\begin{abstract}
The present investigation had as objective to present the existing relations between the motivation for the study and the corporal image of the students belonging to a Private Educational Institution in the district of Santa Anita (Lima-Peru). The research had a correlational descriptive design taking a sample of 210 students of 4 th and 5 th year of secondary education from the same school, to whom two instruments were applied, the Motivation and Learning Strategies Questionnaire and the Questionnaire for the Evaluation of Dissatisfaction with the Body Image (IMAGE). The results reveal that there are significant correlations regarding the motivation to study and body image of the students of the aforementioned Institution.
\end{abstract}

1 Docente en Colegio San Francisco de Borja. Correspondencia. Email: suvero81@hotmail.com

(C) Los autores. Este artículo es publicado por la Revista de Investigación en Psicología de la Facultad de Psicología, Universidad Nacional Mayor de San Marcos. Este es un artículo de acceso abierto, distribuido bajo los términos de la licencia Creative Commons Atribucion - No Comercia_Compartir Igual 4.0 Internacional. (http://creativecommons.org/licenses/by-nc-sa/4.0/) que permite el uso no comercial, distribución y reproducción en cualquier medio, siempre que la obra original sea debidamente citada. 
The dimensions of Motivation and Learning Strategies are mostly related to the factors of

Evaluation of Dissatisfaction with Body Image, except for the dimension Internal attribution of success that is not related.

Keywords: Students; private educational institution; motivation; satisfaction with body image.

En especial los que somos pertenecientes al siglo anterior y vivimos en el siglo XXI podemos dar testimonio de cómo la globalización se ha adentrado en nuestro modo de vivir como sociedad y es que los medios de comunicación influyen distintamente en cada persona. Son los niños y los adolescentes los más afectados por el hecho de que están en plena formación de su personalidad y estas experiencias que perciben del exterior cuando no son clarificadas por el adulto responsable, puede generar dificultades en su sano desarrollo personal.

Los medios de comunicación social nos presentan la llamada cultura de la delgadez, en que mujeres delgadas y varones musculosos se convierten en un estereotipo de hermosura, como si fuese propio de la perfección estética. Los programas de televisión más vistos en la televisión peruana, muestran a jovencitas delgadas o con medidas "perfectas", algunas ayudadas por el bisturí, que se les ve contentas, exitosas y anheladas. Al igual la imagen del varón con musculatura definida, aunque muchos recurren también a otros aditivos.

Hoy en día, se ha incrementado los procedimientos quirúrgicos en más mujeres jóvenes. Siendo cada vez más frecuente, que adolescentes recurran a intervenciones estéticas para aumentar su autoestima, algunas por ser víctimas del bullying. (Paan, 2016). Incluso algunas adolescentes como regalo de quince años o graduación piden a sus padres una lipoescultura o una rinoplastía.

Las razones por las que muchos adolescentes piensan en la cirugía, es para corregir algunas zonas que consideran desagradables (que a veces se convierten en bromas pesadas de parte de sus coetáneos) y ello guarda relación con su imagen corporal.

El adolescente tanto varón como mujer, pasa por distintos cambios hormonales que requieren adaptación y aceptación a su cuerpo. Eresta (2013) afirma que el esquema que uno tiene de su cuerpo, es decisivo en la formación del auto-concepto, el cual se forma con actitudes, comportamientos y motivaciones concretas. Como parte de la definición de su identidad, el adolescente ira reconociendo sus gustos por otras personas y el querer que otros también se fijen en él. Esto sería el comienzo de sentirse a gusto con sí mismo.

En este proceso pueden sentirse vulnerables ante la opinión del resto. La imagen corporal es lo que proyectará a los demás, es lo que los demás verán y según su mente adolescente, lo aceptaran o no, lo catalogaran o no. Para Vaquero-Cristóbal, Alacid, Muyor y López-Miñarro (2013) el esquema corporal 
no siempre se relaciona con las formas reales de su cuerpo, pues influyen las actitudes y valoraciones que la persona tiene de sí mismo. Es decir, la valoración o desestimación que la persona hace de sí mismo influye en su comportamiento ante la sociedad, porque una persona con una alta y estable autoestima puede desenvolverse sanamente en la sociedad siendo un aporte para ella. Para el autor hay más adolescentes insatisfechos con la imagen que perciben de su cuerpo que satisfechos.

Se hace más continua la búsqueda de privacidad con sus amigos y decrece los tiempos para la familia. En estas relaciones con sus pares, pueden darse cuenta que dentro de su grupo de compañeros hay quienes se han desarrollado más físicamente y quizá ello es más notorio para el sexo opuesto, lo que genera un deseo de querer también ser admirado(a). Es por eso que hoy en día en los gimnasios también están yendo los adolescentes sin distinción de género. Siguiendo la línea de los modelos de la televisión, los mismos adolescentes varones ya buscan depilarse las cejas y estilizarse con cortes de cabello. Esa es una parte, pues también se preocupan de lo que comen, por el acné, propios de la edad y por las subidas de peso que evitan para no ser encasillados como "gorditos".

Todos estos pensamientos abordan al adolescente, quien está relacionándose con su cuerpo de un modo diferente de cuando era niño(a), por eso sus estudios y la motivación que tiene por estudiar puede verse comprometida.

Cazalla (2013) sostiene que "El rendimiento del estudiante depende tanto de la capacidad real como de la capacidad creída o percibida. Un autoconcepto positivo proporciona mayor ilusión, mayor capacidad de esfuerzo, mayor resistencia a la frustración en caso de fracaso y mejores resultados académicos..."

Entonces la conducta que el estudiante tiene frente a sus estudios estará influenciada por las actitudes, apreciaciones y aspiraciones, que el mismo tenga de su persona frente a los estudios.

El objetivo clave de esta investigación es señalar las posibles relaciones de la motivación al estudio y la imagen corporal en adolescentes para contribuir concientizando a la comunidad educativa que para los adolescentes el esquema corporal afecta los distintos aspectos donde se desenvuelven, como en el ámbito de los estudios. Al crear conciencia se puede mejorar las relaciones consigo mismo de los adolescentes y mejorar los resultados en sus estudios.

Las hipótesis de la investigación son:

- Existe una relación significativa y positiva entre la variable motivación al estudio y la dimensión Insatisfacción cognitivo emocional de los adolescentes de 4to y 5to de secundaria de una Institución Educativa Privada de Santa Anita. 
- Se encuentra una relación significativa y positiva entre la variable motivación al estudio y la dimensión Insatisfacción perceptiva de los adolescentes de 4to y 5to de secundaria de una Institución Educativa Privada de Santa Anita.

- Existe una relación significativa y positiva entre la variable motivación al estudio y la dimensión Insatisfacción comportamental de los adolescentes de 4to y 5 to de secundaria de una Institución Educativa Privada de Santa Anita.

\section{MÉTODO}

Esta investigación está dentro del tipo no experimental por la razón de que los hechos que se investigan se han generado cómo se han dado realmente. Se caracteriza porque describe y mide diferentes aspectos propios de este estudio con la finalidad de analizarlos y mostrar resultados reales. Este estudio buscó medir el nivel de relación entre dos variables para una situación concreta.

El diseño que muestra la investigación es de forma descriptivo correlacional. Este diseño describe las relaciones entre dos o más variables en un tiempo concreto. Por consiguiente, los diseños correlacionales se limitan a fijar relaciones entre variables sin sostener sentido de causalidad. (Hernández, Fernández y Baptista, 2010)

El universo de esta investigación corresponde a 450 estudiantes de secundaria pertenecientes a una Institución Educativa Privada del distrito de Santa Anita. La muestra de estudio está formada por los 210 alumnos de 4to y 5to de secundaria del mismo colegio. Para seleccionar la muestra se realizó el método no probabilístico intencional pues se determinó de manera voluntaria el distrito, colegio y edad de los adolescentes, teniendo en cuenta la accesibilidad a la muestra y eligiendo éstos dos últimos grados de estudios secundarios, para lograr un mejor resultado y consistencia al no estar en el límite de la edad que corresponde al instrumento.

\section{ANALISIS DESCRIPTIVO GENERAL DE LAS VARIABLES.}

\section{a. MOTIVACION AL ESTUDIO}

Diariamente por distintos factores nos sentimos motivados a realizar nuestros deberes o gustos; incluso en cada decisión hay una motivación que se moviliza por dentro. Por eso podemos decir que la motivación son los estímulos que movilizan a los individuos a efectuar una acción. También puede conceptualizarse como un conjunto de procesos que llevan a que se active, canalice y se mantenga una conducta. (García, 2002). 
Según García, entonces, la motivación hace que la persona para satisfacer una necesidad tenga el impulso necesario para llevarlo a cabo. O en otras palabras como lo expresa Schunk, la motivación es como un mecanismo intrínseco que inicia, conduce y hace que permanezca una conducta. (Gálvez, 2006)

La motivación escolar es un movimiento interno que hace que el estudiante se desarrolle óptimamente o no frente a la educación que recibe. Son las sensaciones, imágenes y perspectivas que tiene de uno mismo, frente a las materias que tiene que aprender, ante las tareas que tiene que realizar, las calificaciones que está obteniendo y también la imagen de estudiante que tienen los otros sobre él.

Existe motivación intrínseca y extrínseca. La primera se refiere a la voluntad del alumno, el cual principalmente quiere estudiar, cuyos intereses pueden ser personales profesionales o escolares, y que le lleva a sacar el empuje de sí mismo, esfuerzo que lo conduce al estudio. (Ramo, 2003). Los alumnos que se interesan por conocer, aprender, que gustan de los retos, son impulsados por la motivación intrínseca porque requiere un interés de la persona. La motivación extrínseca, es el tipo de motivación que el impulso hacia la acción lo hace determinado por circunstancias externas, es decir a través de incentivos negativos o positivos externos al propio individuo y actividad.

\section{b. SATISFACCIÓN CON LA IMAGEN CORPORAL}

La imagen corporal es la representación mental que cada persona tiene de su físico, por eso no siempre coincide con la apariencia real del individuo pues influirá las actitudes y valoraciones que tenga de sí mismo. Aquellas personas que, al evaluar su cuerpo, tienen opiniones que no son iguales a la realidad, tendrían una distorsión de su imagen corporal. En la imagen corporal se observan diversas manifestaciones como satisfacción o insatisfacción, preocupación, evaluación cognitiva, ansiedad y aspectos conductuales. Por eso está referida a la forma como cada ser humano se siente, se ve, se proyecta sobre su corporeidad. Es un concepto que hace referencia a la manera en que uno percibe, imagina, siente y actúa respecto a su corporeidad. (Muñoz, 2014). Por lo tanto la imagen corporal no es un concepto establecido e inamovible, si no que tendrá relación con la madurez que vaya adquiriendo el individuo, la aceptación a su cuerpo real y la relación con éste, sanamente.

Para la constitución del esquema corporal hay dos elementos: la importancia de la Imagen corporal para la autoestima y la satisfacción o la insatisfacción con la misma.

La insatisfacción con el mismo cuerpo se da cuando una persona, percibe su cuerpo según sus ideales y no según la realidad que ve. 
La alteración de la propia imagen es relativamente frecuente, pero depende del grado y consecuencia en otras áreas de la vida para que pueda considerarse como enfermedad. Para considerarlo así hay que realizar diagnósticos especializados. (Vaquero et. al, 2013).

\section{INSTRUMENTOS}

Se utilizó el Cuestionario de Motivación y Estrategias de Aprendizaje, diseñado originalmente por Pintrich en el año 1988. Luego hubieron distintas versiones como de Carlos Luis Ayala Flores.

La escala de motivación tiene 31 ítems y la de estrategias de aprendizaje abarca 50 ítems (31 corresponden a estrategias cognitivas y metacognitivas y el restante se refieren a estrategias de manejo, autorregulación y planificación de recursos). El cuestionario posee diferentes escalas, que fueron creadas para ser más versátil su uso. Para esta investigación hacemos uso de los 31 items primeros, que corresponden a la variable: motivación al estudio. Cuyas dimensiones son las siguientes: Valoración del aprendizaje y del estudio, Motivación intrínseca, Motivación para el trabajo en grupo y para colaborar con los compañeros, Necesidad de reconocimiento, Autoeficacia y Atribución interna del éxito.

Ayala (2014), analiza la fiabilidad de las mediciones a partir de la consistencia interna. Las muestras son adecuadas tanto por su tamaño como por su semejanza con la población de interés. Proporcionan estimaciones del coeficiente alfa y sus intervalos de confianza para cada una de las 10 escalas y para la escala total de Estrategias de Aprendizaje. Los valores de las consistencias aportadas son buenos, aunque hay diferencias en función de las escalas. El rango va de 0.66 a 0.86 , si bien la fiabilidad sube a 0.89 en el caso de la escala total (40 ítems) que evalúa la adecuación de las estrategias de estudio.

También en este estudio se empleó el Cuestionario para la Evaluación de la Insatisfacción con la Imagen Corporal (IMAGEN), cuyos autores son Natalia Solano Pinto y Antonio Cano Vindel (España, 2010). Sus dimensiones son cognitivo-emocional, perceptivo y comportamental y contiene 38 items. Primero se realiza la suma de la puntuación dada por cada persona en cada escala. Las puntuaciones directas deben transformarse en percentiles. La conversión de la puntuación directa en percentil favorece la comparación de las puntuaciones obtenidas acerca de un grupo concreto. Las evidencias de validez se originan por la capacidad de poder medir las evidencias dadas 
por el instrumento empleado. Es decir, es válido cuando las evidencias científicas lo comprueban. Puede haber validez de contenido, constructo y criterio. Los estudios de validación de constructo se refieren a los análisis factoriales exploratorios, los cuales son procesos estadísticos que agrupan los ítems por semejanzas, junto a correlaciones entre las puntuaciones entre los factores que componen las variables trabajadas.

Los estudios psicométricos se realizan sobre dos muestras. La muestra de validación y normativa, la cual aporta los datos para la evaluación de la fiabilidad, validez de constructo y normas; mientras que la muestra de validación cruzada es utilizada para replicar los resultados obtenidos con la primera y la realización de los análisis de validación de criterio.

Los autores del Cuestionario, analizan la fiabilidad de las mediciones a partir de la consistencia interna. Acerca de las muestras empleadas, resultan ser las más convenientes dado a su tamaño y parecido a la población seleccionada. Proporcionan estimaciones del coeficiente alfa y sus intervalos de confianza para cada una de las 10 escalas y para la escala total de Estrategias de Aprendizaje. Los valores de las consistencias aportadas son buenos, aunque hay diferencias en función de las escalas. El rango va de 0.66 a 0.86 , si bien la fiabilidad sube a 0.89 en el caso de la escala total (40 ítems) que evalúa la adecuación de las estrategias de estudio.

\section{RESULTADOS}

Tabla 1

Factores del cuestionario de Motivación y Estrategias de Aprendizaje e Insatisfacción Cognitivo Emocional. Tabulación Cruzada

\begin{tabular}{|c|c|c|c|c|c|}
\hline & & & \multicolumn{2}{|c|}{$\begin{array}{c}\text { Insatisfacción Cognitivo } \\
\text { Emocional }\end{array}$} & \multirow{2}{*}{ Total } \\
\hline & & & $\begin{array}{l}\text { Ni de acuerdo ni } \\
\text { en desacuerdo }\end{array}$ & De acuerdo & \\
\hline \multirow{4}{*}{$\begin{array}{l}\text { Factores de } \\
\text { cuestionario de } \\
\text { Motivación y } \\
\text { Estrategias de } \\
\text { Aprendizaje }\end{array}$} & \multirow{2}{*}{$\begin{array}{l}\text { Ni de acuerdo ni } \\
\text { en desacuerdo }\end{array}$} & Recuento & 7 & 47 & 54 \\
\hline & & Recuento esperado & 12.6 & 41.4 & 54.0 \\
\hline & \multirow{2}{*}{ De acuerdo } & Recuento & 42 & 114 & 156 \\
\hline & & Recuento esperado & 36.4 & 119.6 & 156.0 \\
\hline \multirow{2}{*}{ Total } & & Recuento & 49 & 161 & 210 \\
\hline & & Recuento esperado & 49.0 & 161.0 & 210.0 \\
\hline
\end{tabular}


Tabla 2

Prueba de chi-cuadrado

\begin{tabular}{lccccc}
\hline & Valor & gl & $\begin{array}{c}\text { Sig. asintótica } \\
\text { (2 caras) }\end{array}$ & $\begin{array}{c}\text { Significación } \\
\text { exacta (2 caras) }\end{array}$ & $\begin{array}{c}\text { Significación } \\
\text { exacta (1 caras) }\end{array}$ \\
\hline Chi- cuadrado de Pearson & $4.370^{\mathrm{a}}$ & 1 & .037 & & \\
Corrección de continuidad & 3.625 & 1 & .057 & & \\
Razón de verosimilitud & 4.783 & 1 & .029 & & .025 \\
Prueba exacta de Fisher & & & & .040 & \\
Asociación lineal por lineal & 4.349 & 1 & .037 & & \\
N de casos válidos & 210 & & & & \\
\hline
\end{tabular}

a. $\quad 0$ casillas $(0 \%)$ han esperado un recuento menor que 5. El recuento mínimo esperado es 12.60.

b. Sólo se ha calculado para una tabla $2 \times 2$

Dado que el Chi Cuadrado calculado (4.37) es mayor que el Chi cuadrado crítico (3.841) se rechaza la hipótesis nula y se concluye que los factores del cuestionario de Motivación y Estrategias de Aprendizaje se relacionan en la dimensión insatisfacción cognitivo emocional. Dándonos a conocer que la conducta motivada que los adolescentes que deberían tener para estudiar, es influenciada por sus deseos o necesidades de tener un cuerpo más delgado, o el querer realizar una dieta por sentir malestar emocional en relación a su aspecto físico.

Tabla 3

Factores de cuestionario de Motivación y Estrategias de Aprendizaje e Insatisfacción Perceptiva. Tabulación Cruzada

\begin{tabular}{|c|c|c|c|c|c|c|}
\hline & & & \multicolumn{2}{|c|}{ Insatisfacción Perceptiva } & \multirow{2}{*}{$\begin{array}{c}\text { Total } \\
\text { mente de } \\
\text { acuerdo }\end{array}$} & \multirow[b]{2}{*}{ Total } \\
\hline & & & $\begin{array}{l}\text { Ni de acuerdo ni } \\
\text { en desacuerdo }\end{array}$ & De acuerdo & & \\
\hline \multirow{4}{*}{$\begin{array}{l}\text { Factores de } \\
\text { cuestionario de } \\
\text { Motivación y } \\
\text { Estrategias de } \\
\text { Aprendizaje }\end{array}$} & \multirow{2}{*}{$\begin{array}{l}\text { Ni de } \\
\text { acuerdo ni en } \\
\text { desacuerdo }\end{array}$} & Recuento & 6 & 44 & 4 & 54 \\
\hline & & Recuento esperado & 13.9 & 38.8 & 1.3 & 54.0 \\
\hline & \multirow{2}{*}{ De acuerdo } & Recuento & 48 & 107 & 1 & 156 \\
\hline & & Recuento esperado & 40.1 & 112.2 & 3.7 & 156.0 \\
\hline \multirow{2}{*}{ Total } & & Recuento & 54 & 151 & 5 & 210 \\
\hline & & Recuento esperado & 54.0 & 151.0 & 5.0 & 210.0 \\
\hline
\end{tabular}

Tabla 4

Prueba de chi-cuadrado

\begin{tabular}{lccc}
\hline & Valor & gl & Sig. asintótica (2 caras) \\
\hline Chi- cuadrado de Pearson & $14.669^{\mathrm{a}}$ & 2 & .001 \\
Razón de verosimilitud & 14.517 & 2 & .001 \\
Asociación lineal por lineal & 12.307 & 1 & .000 \\
N de casos válidos & 210 & & \\
\hline
\end{tabular}

a. 2 casillas $(33.3 \%)$ han esperado un recuento menor que 5. El recuento mínimo esperado es 1.29 
Dado que el Chi Cuadrado calculado (14.669) es menor que el Chi cuadrado crítico (5.991) se rechaza la hipótesis nula y se concluye que los factores del cuestionario de Motivación y Estrategias de Aprendizaje se relacionan con la dimensión insatisfacción perceptiva, ya que la motivación que el estudiante tenga para su propio aprendizaje tiene un papel importante para obtener favorables resultados en su vida escolar, por lo tanto al tener una creencia o sensación subjetiva y negativa sobre su propio cuerpo, puede verse afectado en su rendimiento académico.

\section{Tabla 5}

Factores de cuestionario de Motivación y Estrategias de Aprendizaje e Insatisfacción Comportamental. Tabulación Cruzada

\begin{tabular}{|c|c|c|c|c|c|}
\hline & & & \multicolumn{2}{|c|}{ Insatisfacción Comportamental } & \multirow[b]{2}{*}{ Total } \\
\hline & & & $\begin{array}{c}\text { Ni de acuerdo ni } \\
\text { en desacuerdo }\end{array}$ & De acuerdo & \\
\hline \multirow{4}{*}{$\begin{array}{l}\text { Factores de } \\
\text { cuestionario de } \\
\text { Motivación y } \\
\text { Estrategias de } \\
\text { Aprendizaje }\end{array}$} & \multirow{2}{*}{$\begin{array}{l}\text { Ni de acuerdo ni } \\
\text { en desacuerdo }\end{array}$} & Recuento & 2 & 52 & 54 \\
\hline & & Recuento esperado & 6.2 & 47.8 & 54.0 \\
\hline & \multirow{2}{*}{ De acuerdo } & Recuento & 22 & 134 & 156 \\
\hline & & Recuento esperado & 17.8 & 138.2 & 156.0 \\
\hline \multirow{2}{*}{ Total } & & Recuento & 24 & 186 & 210 \\
\hline & & Recuento esperado & 24.0 & 186.0 & 210.0 \\
\hline
\end{tabular}

Tabla 6

Prueba de chi-cuadrado

\begin{tabular}{lccccc}
\hline & Valor & gl & $\begin{array}{c}\text { Sig. asintótica } \\
\text { (2 caras) }\end{array}$ & $\begin{array}{c}\text { Significación } \\
\text { exacta (2 caras) }\end{array}$ & $\begin{array}{c}\text { Significación } \\
\text { exacta (1 cara) }\end{array}$ \\
\hline Chi- cuadrado de Pearson & $4.285^{\mathrm{a}}$ & 1 & .038 & & \\
Corrección de continuidad & 3.320 & 1 & .068 & & \\
Razón de verosimilitud & 5.224 & 1 & .022 & & .027 \\
Prueba exacta de Fisher & & & & .046 & \\
Asociación lineal por lineal & 4.265 & 1 & .039 & & \\
N de casos válidos & 210 & & & & \\
\hline
\end{tabular}

a. 0 casillas $(0.0 \%)$ han esperado un recuento menor que 5. El recuento mínimo esperado es 6.17.

b. Sólo se ha calculado para una tabla $2 \times 2$

Dado que el Chi Cuadrado calculado (4.285) es mayor que el Chi cuadrado crítico (3.841) se rechaza la hipótesis nula y se concluye que los factores del cuestionario de Motivación y Estrategias de Aprendizaje se relacionan en la dimensión insatisfacción comportamental. Lo que nos dice que su vida académica puede verse perjudicada si tiene comportamientos insanos contra su propio cuerpo 
como inducirse el vómito, tomar laxantes, botar comida, realizar excesivamente ejercicio físico; lo que evidentemente afectará su rendimiento.

\section{DISCUSIÓN}

\section{- Respecto a la Motivación al Estudio}

Acerca de esta dimensión y la Valoración del Aprendizaje y del Estudio, los estudiantes resaltan estar de acuerdo ante las opciones de priorizar el estudio antes que el trabajo, lo que guarda relación con Alvites (2012) quien sustentó que desde la perspectiva psicológica, aquella persona que está motivada para el estudio, comprende que el conocimiento es necesario para desenvolverse óptimamente en la vida.

Se ha encontrado con respecto a la Motivación al Estudio en la dimensión Motivación Intrínseca en los ítems del cuestionario, que los estudiantes manifestaron ni estar en acuerdo ni en desacuerdo con respecto al incrementar sus conocimientos con repaso o investigación de temas. También manifiestan sentirse de acuerdo en relación a una adecuada motivación hacia el estudio, relacionándose con Toapanta (2012) quien afirma que cuando uno determina su motivación por el estudio o trabajo está dando a conocer que desea superarse y que tiene una personalidad que le ayuda en la realización de sus aspiraciones y objetivos.

Los principales hallazgos que se han mostrado con respecto a la Motivación al estudio en la dimensión Motivación para el Trabajo en Grupo y para Colaborar con los Compañeros manifestaron en su mayoría de respuestas estar "ni en acuerdo ni en desacuerdo" en relación a sentirse estimulados al trabajo escolar con otros compañeros. Sobresale la alternativa marcada sobre el gusto de elegir la forma de trabajar, individual o grupalmente, ya que ello enriquece el producto final que se busca, como lo expresa Ruiz (2012) quien en su tesis propone, desarrolla y examina los resultados y logros de aplicar un sistema de cooperación mostrando que el aprendizaje colaborativo aumenta cualitativamente el aprendizaje y potencia habilidades personales que requieren la motivación del individuo.

Referente a la Motivación al Estudio y la dimensión Necesidad de reconocimiento se ha hallado que la mayoría de respuestas están asociadas a estar "ni en acuerdo ni en desacuerdo" con la opinión que ejerce su entorno cercano sobre el ámbito educativo que lo envuelve. Navea (2015) afirma que el estudiante le favorece el reconocimiento que proporciona una retroalimentación informativa que beneficia su aprendizaje, utilizando recompensas o incentivos, con los hallazgos en las respuestas de los estudiantes muestran que no dependen del reconocimiento de sus compañeros o docentes sobre sus logros académicos, que si bien son importantes las opiniones o expectativas de los otros, no son determinantes. 
En lo concerniente a Motivación al Estudio se encontraron importantes hallazgos en la mayoría de los ítems referentes a la dimensión Autoeficacia, relacionado a sentirse con la capacidad de responder en los estudios. Demostrando el estar ni en acuerdo ni en desacuerdo con la impresión que cada uno tiene de sí mismo para alcanzar ciertas metas, frente a otras respuestas; como sustenta la tesis presentada por Barrientos (2011), quien sostiene que la motivación cuando está relacionada con la autoeficacia, está referida a las experiencias anteriores que tenga el individuo y que lo lleva a su accionar frente a los problemas.

Respecto a la Motivación al Estudio en la dimensión Atribución Interna del Éxito, la mayoría manifestaron estar de acuerdo de que si alcanzamos las metas dependerá de uno mismo como Flores (2010) los estudiantes serán exitosos cuando consideran que para tener éxito en la vida debe darse por causas internas suyas como son las habilidades y aptitudes.

\section{- Respecto a la Imagen Corporal}

En lo referente a Imagen Corporal se encontraron importantes hallazgos con lo que respecta a la dimensión Insatisfacción Cognitivo-emocional en los ítems relacionados a la opinión del entorno sobre el cuerpo de otra persona. Colocando la mayoría de estudiantes, la alternativa "de acuerdo" o "totalmente de acuerdo" a las valoraciones sobre su cuerpo, que hacen los demás; tal como se refiere Sánchez (2012), quién afirmó que actualmente hay una presión social que introduce un mensaje de cómo debe ser el cuerpo ideal, dándole la idea de que solo si se consigue tener un cuerpo perfecto, se logrará el éxito tanto en lo personal como en lo profesional.

Se ha encontrado en relación al esquema corporal y a la dimensión Insatisfacción perceptiva, que la mayoría de estudiantes colocaron estar de acuerdo con el modo en que perciben su cuerpo, con desagrado y /o desestimándolo; lo que guarda relación con la tesis de Ortega (2012), encontrando diferencias significativas en la percepción de la figura que desean tener y no en el IMC real de los estudiantes de primer ciclo de Educación Secundaria en Ciudad de Jaén (ESPAÑA), lo que sugiere que los estudiantes se consideran diferente a como se da a conocer en los datos obtenidos del Índice de Masa Corporal .

Los hallazgos en la variable de estudio Imagen corporal en relación a la dimensión Insatisfacción comportamental, dan a conocer que la mayoría toma acciones a partir de la percepción que tienen de su cuerpo. Así en la tesis de Enríquez (2015) concluye con su investigación en adolescentes de la Institución Educativa "Hosanna" (Miraflores) que los adolescentes, influenciados por padres y/o amigos, tratan de mejorar su imagen física a través de dietas, ejercitándose y empleando medicina con el deseo de disminuir su peso o aumentar su estatura. 
Estos hallazgos ayudan a toda la comunidad científica para posteriores estudios relacionados con las variables trabajadas en esta investigación. También puede dar claridad para comprender que los estudiantes de 4to y 5 to de secundaria que manifiestan insatisfacción comportamental, perceptiva y/o cognitivo emocional, tendrán dificultades en su desempeño académico pues su motivación para el estudio estará afectada.

\section{CONCLUSIONES}

- A través de este estudio se establecieron vínculos significativos respecto a la motivación al estudio y la dimensión Insatisfacción cognitivo emocional de los adolescentes de 4to y 5to de secundaria de una Institución Educativa Privada de Santa Anita.

- Con este estudio se establecieron vínculos significativos acerca de la motivación al estudio y la dimensión Insatisfacción perceptiva de los adolescentes de 4to y 5to de secundaria de una Institución Educativa Privada de Santa Anita.

- En esta investigación se establecieron vínculos significativos respecto a la motivación al estudio y la dimensión Insatisfacción comportamental de los adolescentes de 4to y 5to de secundaria de una Institución Educativa Privada de Santa Anita.

\section{REFERENCIAS}

Alvites, J. (2012). Relación entre los grados de motivación para el estudio y los grados de valores morales en postulantes al SENATI. Tesis de maestría inédita. Universidad Nacional Mayor de San Marcos, Lima-Perú.

Ayala, C. (2004). Cuestionario de estrategias de aprendizaje y motivación. Madrid: Editorial EOS.

Barrientos, L. (2011). Motivación Escolar y Rendimiento Académico en alumnos del cuarto año de secundaria de una Institución Educativa Estatal de Ventanilla. Tesis de maestría inédita. Universidad San Ignacio de Loyola, Lima-Perú.

Cazalla-Luna, N. (2013). Revisión teórica sobre el autoconcepto y su importancia en la adolescencia. Recuperado de https://revistaselectronicas.ujaen.es/index.php/reid/ article/viewFile/991/818

Enríquez, R. (2015). Investigación sobre la autopercepción de la imagen corporal y prácticas para corregirla en adolescentes de una institución educativa. Lima: Universidad Nacional Mayor de San Marcos.

Eresta, M. (2013). Relaciones afectivas y sexualidad en la adolescencia. Madrid: Liga Española de la Educación. 
Flores, R. (2010). Un estudio sobre la motivación hacia la escuela secundaria en estudiantes mexicanos. México: Universidad Nacional Autónoma de México.

Gálvez, A. (2006). Motivación hacia el estudio y la cultura escolar. Pensamiento Psicológico, Vol.2, $\mathrm{N}^{\circ} 6$, p. $87-101$.

García, F. (2002). Motivación, aprendizaje y rendimiento escolar. Revista electrónica de Motivación y emoción, Vol.1, $\mathrm{N}^{\circ}$ 0. Recuperado de http://reme.uji.es/articulos/ pa0001/texto.html

Hernández, R., Fernández, C. y Baptista. M. (2010). Metodología de la investigación. México: Mc Graw Hill.

Muñoz, A.M. (2014) La imagen corporal en la sociedad del siglo XXI. Universidad Internacional de Catalunya, España

Navea, A. (2015). Un estudio sobre la motivación y estrategias de aprendizaje en estudiantes universitarios de Ciencias de la Salud. Tesis para optar Doctorado en Investigación e Innovación en Diagnóstico y Orientación Educativa. UNED. Universidad Nacional de Educación a Distancia, España.

Ortega, M. (2012). Relación entre la insatisfacción con la imagen corporal, autoestima, autoconcepto físico y la composición corporal. Tesis para el Programa de Doctorado: Actividad física para la educación en la sociedad del conocimiento. Editorial de la Universidad de Granada, España.

Paan, C. (2016). Cirugía estética: Adolescentes son el nuevo gran mercado. El Comercio. Recuperado de https://elcomercio.pe/economia/dia-1/cirugia-estetica-adolescentesson-nuevo-gran-mercado-227736

Ruiz, D. (2012). La influencia del trabajo cooperativo en el Aprendizaje del área de economía en la Enseñanza secundaria. Tesis doctoral inédita Universidad de Valladolid, España.

Ramo, A. (2003). La Motivación en el estudio. Especial desde España para El Cronista Regional, 19/12/03.

Sánchez, I. (2012). Insatisfacción corporal en hombres y mujeres de población rural. Recuperado de http://www.academia.edu/3640121/Tesis_insatisfaccion_corporal

Toapanta, S. (2011). La Motivación en el aprendizaje de los estudiantes de cuarto año de Educación Básica, de la Unidad Educativa Fiscal Mixta "Brethren”, Parroquia Calderón, Cantón Quito. Proyecto socio-educativo presentado para Optar el Grado de Licenciatura en Ciencias de la Educación. Universidad Central de Ecuador, QuitoEcuador.

Vaquero-Cristóbal, R, Alacid, F, Muyor, J. y López-Miñarro, P. (2013). Imagen corporal. Nutrición hospitalaria, 28 (1). Recuperado de http://dx.doi.org/10.3305/ nh.2013.28.1.6016. 
